\title{
Reconstruction of the charged surface of liquid hydrogen
}

\author{
G. V. Kolmakov, A. A. Levchenko, L. P. Mezhov-Deglin, and A. B. Trusov \\ Institute of Solid State Physics, RAS, 142432, Chernogolovka, Moscow District, Russia, \\ E-mail: levch@issp.ac.ru
}

\begin{abstract}
The evolution of shape of the surface of equipotentially charged liquid hydrogen film condensed on the lower or upper plate of a horizontally placed diode in external electric fields has been studied experimentally under the condition of total compensation of the applied field by the surface charge. Reconstruction phenomenon - the formation of a solitary wave (soliton) - has been observed in an electric field higher than some critical value for the film that covers the lower plate.
\end{abstract}

PACS: 68.10.-m

\section{Introduction}

It is known that a charged flat surface of a liquid becomes unstable in an external, perpendicular, electric field $E$ that exceeds a critical value [1]. Until the time we began this study the reconstruction of a negatively charged surface of a thick layer of liquid helium (electrons above the helium) was studied experimentally and theoretically in detail in the case where the liquid depth $d$ (the distance from the free surface to the metallic control substrate placed in its interior) exceeded noticeably the capillary length, $\lambda=\sqrt{\alpha / \rho g}$. Here $\alpha$ and $\rho$ are the surface tension and the density of liquid helium, and $g$ is the free fall acceleration. The theoretical considerations were restricted to the case where the electrons that are localized above the surface do not compensate for the applied electric field. It was found that for $d>>\lambda$ and a fixed number of charges above the surface, when the field exceeds the critical value, a periodic deformation, which is accompanied by the surface charge density modulation with a period of the order of $\lambda$ and finite amplitude (equal to $\lambda$ in order of magnitude), arises on the surface of the liquid (dimple crystal) [2-4]. This phenomenon was studied experimentally in Refs. 5-7.

In the opposite case, at $d<<\lambda$ the range of the wave numbers in which the instability develops starts at $k=0$ (Ref. 8). Theoretical investigations [9] show that in fields higher than the critical field the stationary structure can also appear on the charged surface, but the character of the reconstruction differs markedly from the previuos situation: the amplitude of the deformation is proportional to the supercriticality level, while the period is inversly proportional to the supercriticality. Thus, at fields slightly exceeding the threshold value the amplitude of the structure is small with respect to the capillary length $\lambda$.

Theoretical estimates performed for the charged surface of liquid helium layer that has infinite horizontal dimensions and finite thickness $d>>\lambda$ under the condition of complete compensation have shown that reconstruction to the steady state is impossible, and that the periodic discharge from the surface has to take place.

Until recently it was not clear whether the reconstruction can be observed on the surface under the conditions that the distance $d$ between the control electrode placed above the liquid and the charged liquid surface is nearly equal to $\lambda$, and the electric field is completely compensated for by charges localized below the surface. Preliminary results of our experiments were published in Ref. 10.

\section{Experimental procedure}

The experiments were performed using two different diodes D1 and D2 inserted into an optical container. As the charge source we used radioactive plates emitting $\beta$ electrons of 5 - $\mathrm{keV}$ mean energy. The electrons ionized a layer of liquid about $10 \mu \mathrm{m}$ thick near the plate.

In the diode D1 we used a metallic guard ring $25 \mathrm{~mm}$ or $3 \mathrm{~mm}$ inside diameter and $3 \mathrm{~mm}$ height. This ring prevented the charges from escaping from the surface of the liquid to the walls of the con- 
tainer. The guard ring and the radioactive source formed a cup in which the hydrogen was condensed. A metallic collector $25 \mathrm{~mm}$ in diameter (control electrode) was placed above the radioactive source. The distance from the source to the collector was 6 $\mathrm{mm}$.

In the diode D2 a 3-mm-diameter charge source was placed on the upper copper plate of the diode, on which the hydrogen was condensed. The sourceto-lower plate distance was $3 \mathrm{~mm}$. The sign of the charges on the surface of the liquid was dictated by the polarity of the applied voltage $U$. The properties of the positively charged surface were investigated experimentally. The shape of the charged surface was controlled visually with a TV camera, and a change in the curvature of the charged surface was determined from the deflection of the laser beam, which was reflected from the surface.

\section{Results}

At small voltages between the diode plates the equipotentially charged surface of liquid wetting lower plate of the diode D1 is curved smoothly. Once a certain critical voltage $U_{c 1}$ is reached, a macroscopic hump appears with a characteristic diameter of several millimeters (Fig. 1). It should be noted that the reconstructed region could occur at the random sites of the surface. In the runs the solitary hump could be shifted toward the diode center by slightly changing the tilt of the cell.

The height of the deformed surface region (hump) at the diode center could be controlled by changing the voltage between the plates within $V=1300-1700 \mathrm{~V}$. At voltages $U$ below the threshold voltage ( $U_{c 1} \approx 11300 \mathrm{~V}$ at the given temperature) no surface reconstruction was observed. As the voltage was increased to $1750 \mathrm{~V}$, the reconstructed surface lost its stability, and a discharge pulse was observed (second critical voltage $U_{c 2}$ ). In the course of a discharge about $10^{8}$ charges arrive at the collector. After the discharge the surface relaxes to the original flat state and then the process is repeated. We have reported earlier on our observa-

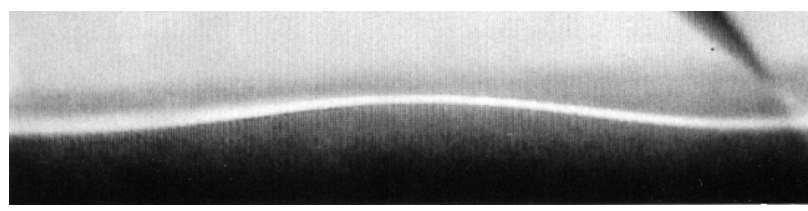

Fig. 1. Profile of the charged surface of liquid hydrogen at $U=1620 \mathrm{~V}$ and $T=17 \mathrm{~K}$. The «hump» is clearly seen at the center. The linear dimension of the photograph in the horizontal position is $10 \mathrm{~mm}$.

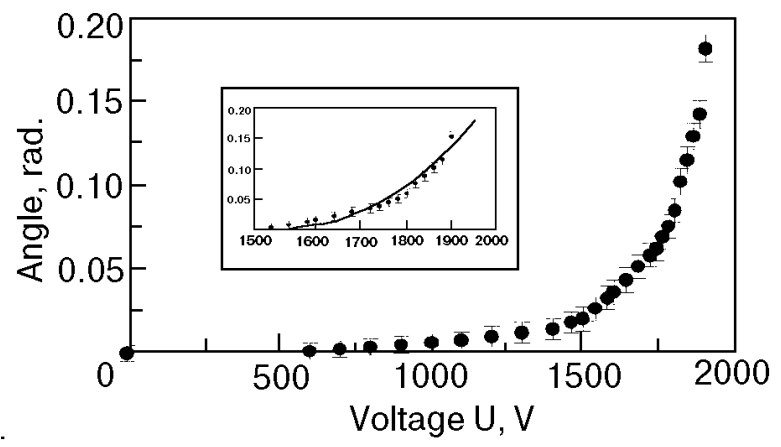

Fig. 2. Field dependence of the laser beam maximal reflection angle from the surface of the $\ll$ hump» at $T=14.6 \mathrm{~K}$.

tions of quasiperiodic oscillations of a charged surface of liquid hydrogen [11]. An analogous phenomenon - the occurrence of ionic jets (geysers) on the surface of superfluid helium in strong nonuniform fields - was observed in Ref. 12.

Figure 2 illustrates the voltage dependence of the maximal value of the reflection angle $\beta$ of the laser beam from the surface of liquid measured at the temperature of $14.6 \mathrm{~K}$. The dependence of the reflection angle on the control voltage $\beta(U)$ was found to change considerably at voltages in excess of the first critical voltage (in these measurements $U_{c 1} \approx 1500 \mathrm{~V}$ ).

Figure 3 shows another situation: the equilibrium profile of the film wetting the upper plate of the diode D2 at a voltage $U=1300 \mathrm{~V}$ between the plates and temperature $T=15 \mathrm{~K}$.

It should be emphasized that the volume of the liquid film does not change with increasing voltage. When the voltage increases, the shape of the film transforms gradually from a flat film to a new drop-like film. The height of the droplet increases and the visible diameter of its base desreases. At a voltage above $2000 \mathrm{~V}$ a pulse discharge is been observed.

Figure 4 shows the voltage dependence of the height of the droplet. In contrast to the dependence

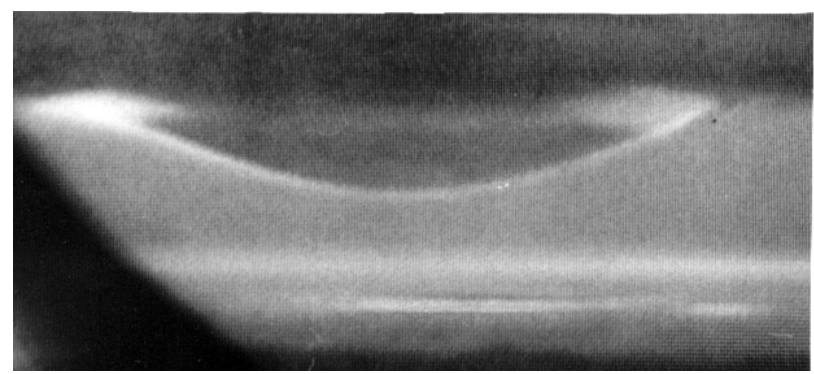

Fig. 3. Photographe of the profile of the charged surface of the film condensed on the upper plate of the diode. The voltage $U=1300 \mathrm{~V}, T=15 \mathrm{~K}$ 


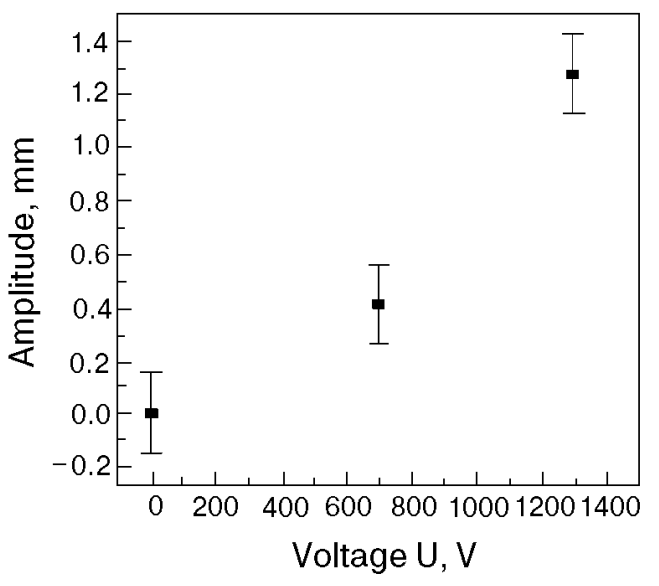

Fig. 4. Field dependence of the height of the droplet created at the upper plate of the diode, $T=15 \mathrm{~K}$

shown in Fig. 2, here we observe a rapid increase of the height at small voltages.

Since in this «inverse» geometry the power of the charge source was not very high, we had to wait for several minutes until the charge density reached its equilibrium value and could compensate completely for the external electric field. This circumstance enabled us to record the evolution of the profile of the surface for the conditions under which the charges did not compensate the applied field.

The shape of the droplet shown in Fig. 5 differs significantly from the profile shown in Fig. 1. Here charges accumulate at the center of the droplet. In Fig. 6 we show the profile of this droplet which was obtained by scanning the photograph in Fig. 5. This situation seems to be analogous to the case of the formation of an individual dimple on the surface of liquid helium with a fixed number of electrons above the surface studied earlier [13]. In a field higher than the critical field (near $2800 \mathrm{~V}$ ) the surface loses its stability and a discharge takes place. This process can be repeated at a constant voltage.

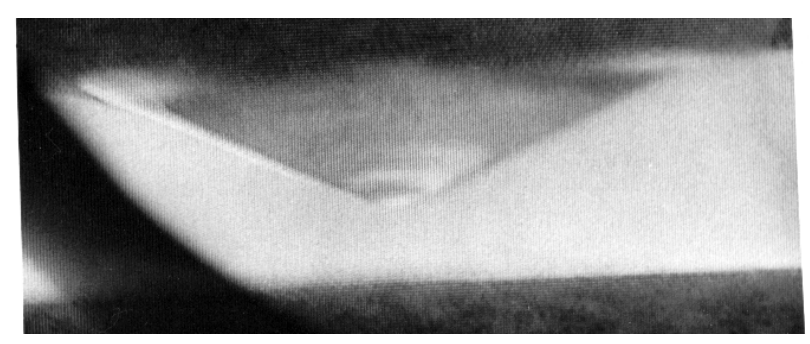

Fig. 5. Profile of the surface of the droplet wetting the upper plate. The charges have accumulated at the center of the droplet. $U=2200 \mathrm{~V}, T=15 \mathrm{~K}$.

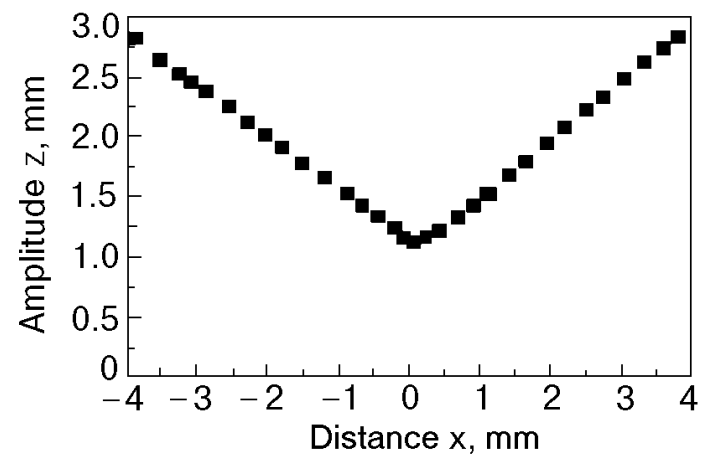

Fig. 6. Profile of the charged surface of the droplet shown in Fig. 5. Plots are the results of scanning.

\section{Discussion}

The experimental situation in our work is qualitatively different from the situation that has been observed in Ref. 13 and discussed in Refs. 3 and 4. In our experiments the charge density is governed by the applied voltage: the charges completely compensate for the external electric field, and their density is equal to the maximum allowable value $n=n_{c}=E^{2} / 4 \pi$, throughout the surface, whereas in Ref. 13 the charge density on the flat surface away from the reconstructed region (dimple) is close to zero, $n<<n_{c}$. In addition, in our case the total number of charges localized near the surface is not fixed and it increases with increasing applied voltage.

The observations show that the lifetime of the reconstructed surface of hydrogen is many times longer than the characteristic time of charging and discharging of the surface. This implies that there exists a stationary charged solitary wave (hump).

The authors of Ref. 9 considered the one-dimensional case, which corresponds to the formation of a periodic system of «rolls» on an infinite surface of a thin helium film $d<<\lambda$ at a field exceeding a critical field. The shape of a solitary roll $(1 D$ soliton), which corresponds to an infinitely long period (for the experiment, much greater than the diameter of the experimental cell), is described by the expression

$$
z(x)=A \cosh ^{-2}(x / R),
$$

where $A$ is the amplitude, and $R$ is the characteristic dimension of the soliton.

Nevertheless, it turned out that the shape of the hump observed in experiments with liquid hydrogen can be described satisfactory by expression (1), provided that the hump is axisymmetric and $x$ is replaced by the distance $r$ from the center of the 


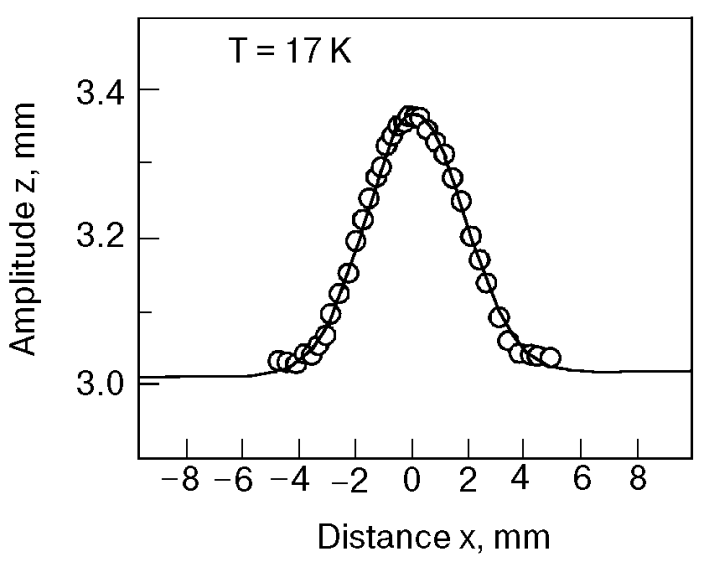

Fig. 7. Profile of the hump, obtained by scanning the photograph shown in Fig. 3 (circles). Solid line - the function (1) with the parameters $A=0.38 \mathrm{~mm}, R=2.5 \mathrm{~mm}$.

soliton. Then $R$ is the characteristic radius of the soliton. Numerical fitting of the profile of the hump yielded the values $A=0.38 \mathrm{~mm}$ and $R=2.5 \mathrm{~mm}$ (the solid curve in Fig. 7).

The evolution of the shape of the hump with increasing applied voltage can be seen from the dependence $\beta(U)$. The plot in Fig. 2 illustrates that there exist two different regimes of alteration of the shape of a free surface of the liquid - below and above the first critical voltage $U_{c 1}$. The initial part $\left(U<U_{c 1}\right.$ ) corresponds to the usual drawing of the charged liquid into the field of the capacitor. In this case the reflection angle is proportional to the square of applied voltage, $\beta \propto U^{2}$. In fields above the first critical field, i.e., in the region $U_{c 1}<U<U_{c 2}$, the experimental dependence can be described by the relation

$$
\beta(U) \propto\left(U^{2} / U_{c 1}^{2}-1\right)^{3 / 2} / U^{2},
$$

which follows from theoretical consideration of the hump on the surface of a thin liquid layer [9]. The insert in Fig. 2 compares the experimental data in fields higher the first critical field with the theoretical dependence $\beta(U)$. This section of the curve $\beta(U)$ describes the change in slope of the lateral surface of the soliton with increasing voltage $U$. Fitting the theoretical curve to the experimental data gives the first critical field, $U_{c 1}=1500 \mathrm{~V}$. The results are found to be in good agreement with the theoretical estimation from Ref. 8.

Lett us now discuss the results of the experiments with a liquid layer covering the upper plate of the diode. Figure 8 illustrates the results of scanning the photograph of the profile of the charged surface of the droplet condensed on the upper plate of the diode, shown in Fig. 3. The

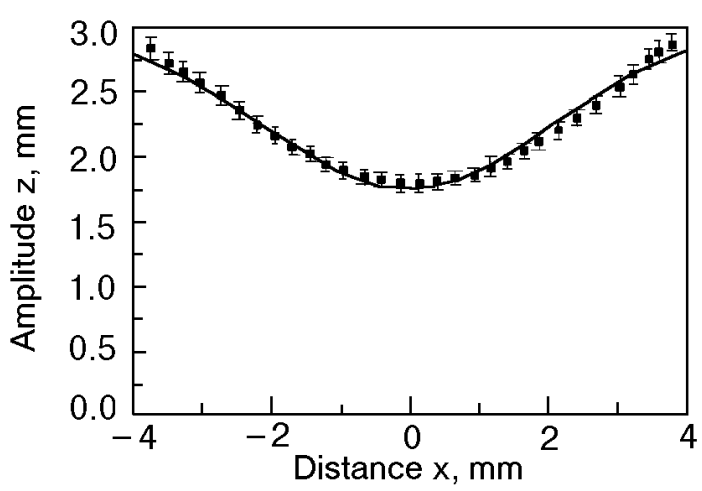

Fig. 8. Profile of the droplet obtained by scanning the photograph shown in Fig. 3. Dark squares - experiment; solid line - theory.

solid curve presents the trial function $f(x)=$ $=A \exp \left(-x^{2} / R^{2}\right) \quad$ with the fitting parameters $A=1.25 \mathrm{~mm}$ and $R=3 \mathrm{~mm}$. The trial function describes satisfactorily the experimental data, and the effective radius $R$ coincides with the capillary length of liquid hydrogen.

\section{Conclusions}

From the results of experiments on the diode D1 (Figs. 1, 2 and 7) we conclude that 1) The reconstruction of a charged surface in a static electric field in a cell of finite diameter at $d \sim \lambda$ is possible at variable number of charges and 2) Under the conditions when the charge density on the equipotential surface is governed by an external field, in fields exceeding the critical value $U_{c 1}$, a solitary stationary wave (hump) appears on the surface. As the field is further increased the impulse discharge is observed; i.e., there exists the second critical field $U_{c 2}$, above which the reconstructed surface of the liquid is unstable.

From the field dependence shown in Fig. 4 it follows that in the case of the film that covers the upper plate of the horizontal diode the first critical field equals to zero and direction of the free fall acceleration affects crucially the evolution of the charged surface shape in the external electric field.

This work was supported in part by grant from NASA-RSA, project No.TM-17.

1. L. D. Landau and E. M. Lifshitz, Electrodynamics of continuous media, Nauka, Moscow (1982).

2. L. P. Gor'kov and D. M. Chernikova, JETP Lett. 18, 68 (1973); DAN USSR, 228, 829 (1976).

3. Mel'nikov V. I. and S. V. Meshkov, Zh. Eksp. Teor. Phyz. 82, 1910 (1982).

4. V. B. Shikin and Yu. P. Monarkha, $2 D$ charge systems in helium, Nauka, Moscow (1989).

5. P. Leiderer, Phys. Rev. B20, 4511 (1979). 
6. M. Wanner and P. Leiderer, Phys. Rev. Lett. 42, 315 (1979).

7. P. Leiderer and M. Wanner, Phys. Rev. Lett. A73, 185 (1979).

8. D. M. Chernikova, Fiz. Nizk. Temp. 2, 1374 (1976).

9. V. B. Shikin and P. Leiderer, Fiz. Nizk. Temp. 23, 2 (1997).

10. A. A. Levchenko, G. V. Kolmakov, L. P. Mezhov-Deglin, V. B. Shikin, E. Teske, and P. Leiderer, Zh. Eksp. Teor. Fiz. 65, 547 (1997).
11. A. A. Levchenko and L. P. Mezhov-Deglin, Fizk. Nizk. Temp. 22, 46 (1996).

12. V. P. Volodin and M. S. Khaikin, Pisma v Zh. Eksp. Teor. Fiz., 30 (9), 608 (1979).

13. P. Leiderer, W. Ebner, and V. B. Shikin, Surface Sci. 113, 405 (1982). 